\title{
O PROBLEMA DE CORTE DE ESTOQUE COM APROVEITAMENTO DE SOBRAS: UM ESTUDO DE COMPARAÇÃO DE DIFERENTES MODELOS MATEMÁTICOS E HEURÍSTICAS DE RESOLUÇÃO
}

\section{THE CUTTING STOCK PROBLEM USING THE WASTE: A STUDY INTO COMPARISON OF DIFFERENT MATHEMATICAL MODELS AND HEURISTCS FOR RESOLUTION}

\author{
Eduardo Aparecido da Rosa Neto ${ }^{1}$; Robinson Samuel Vieira Hoto ${ }^{2}$ \\ ${ }^{1}$ Universidade Estadual de Londrina - UEL \\ eduardo.neto@ensinolivre.com.br \\ ${ }^{2}$ Universidade Estadual de Londrina - UEL \\ hoto@uel.br
}

\begin{abstract}
Resumo
Um Problema de Corte de Estoque (PCE) consiste basicamente em cortar um conjunto de peças maiores (matéria-prima) disponíveis em estoque com a finalidade de produzir um conjunto de peças menores (itens), otimizando um determinado objetivo, que pode ser minimizar a quantidade total de matéria-prima a ser cortada, bem como as sobras geradas pelo processo ou o custo associado ao corte; maximizar o lucro, entre outros. Neste contexto, o Problema de Corte de Estoque com Aproveitamento de Sobras (PCES) enfoca tal questão visando a melhor utilização da matéria-prima, ou seja, utilizar as sobras em um processo de corte futuro, desde que elas apresentem condições para isso. Buscamos com este trabalho determinar soluções inteiras para um PCES por meio de um estudo computacional dos modelos matemáticos propostos por Pinto (2008). Exploramos também uma reformulação do modelo matemático da Estratégia 5 de Pinto (2008), a qual denominamos Estratégia 5 Reformulada (5R). Analisamos a eficácia dos modelos, considerando critérios de avaliação estabelecidos, a priori, pela comparação dos resultados com os resultados de heurísticas propostas por Cherri e outros (2009), por meio de simulações computacionais realizadas em um conjunto de classes de exemplares gerados aleatoriamente. Os resultados mostram que, conforme as classes vão crescendo em termos de dimensão e demanda dos exemplares, a concentração dos melhores resultados tende para a Estratégia $5 R$ e para os procedimentos heurísticos, em particular para os residuais $R A G_{R} 1, R A G_{R} 2$ e $R A G_{R} 3$.
\end{abstract}

Palavras-chave: problema de corte de estoque unidimensional; aproveitamento de sobras; heurísticas; gerador aleatório; problema de programação linear inteira.

\section{Introdução}

As indústrias de manufatura, principalmente indústrias de papel, metalúrgica, vidro, móveis, plástica, têxtil, entre outras, têm sido estimuladas a aprimorar seus processos, tornando-os cada vez mais eficientes e econômicos. Em tais indústrias, as etapas do gerenciamento de produção estão frequentemente associadas à utilização de estratégias de corte de matérias-primas para transformá- 
las em produtos finais, o que têm motivado a comunidade científica nacional e internacional na busca de métodos de solução eficientes. Uma linha de pesquisa importante na área de Pesquisa Operacional e que têm sido estudados por um número crescente de pesquisadores nas últimas décadas é o Problema de Corte de Estoque (PCE). Esse estudo tem gerado contribuições significativas em diversas áreas, principalmente em razão da importância econômica aliada aos avanços nos aspectos computacionais, interesse que pode ser comprovado, por exemplo, pela consulta a livros dedicados exclusivamente ao tema, em centenas de artigos de revisão e edições especiais de revistas nacionais e internacionais disponíveis na literatura, e pela formação de grupos de pesquisa para tratar exclusivamente do tema (p. ex. GPCE, ESICUP ${ }^{1}$ ).

Destacamos alguns artigos que nortearam este trabalho, a citar: Poldi e Arenales (2009), Hoto e outros (2003), Cherri e outros (2009), Wäsher e outros (2007), Cui e Yang (2010), Gilmore e Gomory (1963) e Gau e Wäsher (1995). Centenas de outros artigos podem ser acessadas pelo endereço eletrônico <http://www.fe.up.pt/esicup>.

O problema de corte de estoque unidimensional pode ser descrito da seguinte forma: suponha que tenhamos em estoque uma quantidade suficiente de barras de comprimento $L$ para cortar itens de comprimentos $l_{i} \leq L, i=1, \ldots, I$, de modo a atender demandas $d_{i}, i=1, \ldots, I$. Ver Figura 1. O problema consiste em efetuar o processo de corte, minimizando a sobra de material. Neste trabalho este problema será denominado Problema de Corte de Estoque Unidimensional Clássico (PCEC).

Figura 1 - (a) Conjunto de barras em estoque de comprimento $L$ a serem cortadas;

(b) Conjunto de itens de comprimentos $\ell_{i} \leq L$ e demandas $d_{i}, i=1, \ldots, I$

(a)

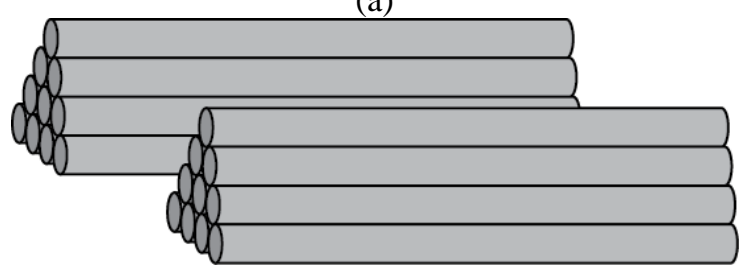

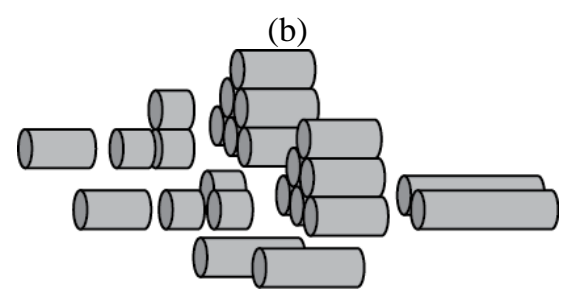

Fonte: Autoria própria (2015)

Por exemplo, uma bobina (de papel, alumínio, tecido, aço,...) atravessa uma máquina com facas, que fará cortes em apenas um sentido (Figura 2).

\footnotetext{
${ }^{1}$ GPCE e ESICUP são grupos (nacional e internacional, respectivamente) que reúnem profissionais, pesquisadores e educadores da Pesquisa Operacional com interesses na área de corte e empacotamento.
} 
Figura 2 - Esquema representando o processo de corte de uma bobina

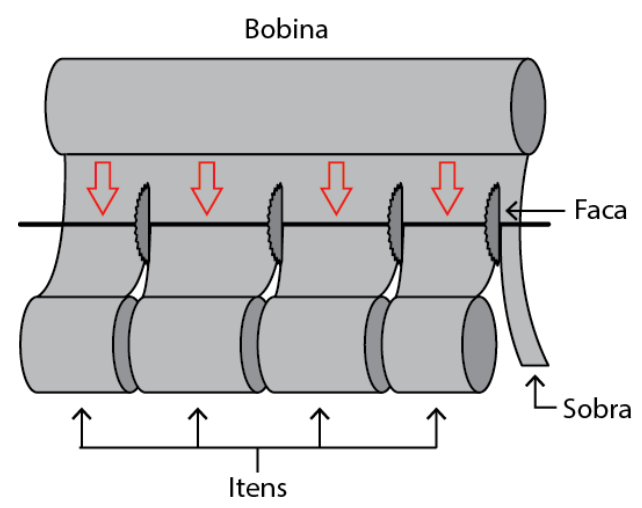

Fonte: Autoria própria (2015)

$\mathrm{Na}$ indústria papeleira, por exemplo, bobinas-jumbo são cortadas em sub-bobinas que, por sua vez, podem ainda ser cortadas em retângulos de diversos tamanhos.

De acordo com o comprimento da bobina, as quantidades e os comprimentos dos itens demandados, há finitas maneiras de arranjos para a disposição das facas, mas esse número pode ser muito grande, mesmo para problemas considerados "pequenos",2. Denotaremos cada disposição das facas no processo de corte de padrão de corte. As variáveis de decisão, neste caso, baseiam-se na utilização de cada padrão de corte, as quais, em razão das restrições de maquinário, por exemplo, devem ser inteiras.

Vamos considerar que há $P$ possibilidades, o que sugere a definição de padrão de corte como um vetor de dimensão $I$ que contabiliza os itens produzidos.

$$
\mathbf{a}_{\mathbf{p}}=\left(a_{1 p}, a_{2 p}, \ldots, a_{i p}, \ldots, a_{I p}\right)^{T}, \quad i=1, . ., I, p=1, . ., P
$$

Em que $\mathbf{a}_{\mathrm{p}}$ é o vetor representante do p-ésimo padrão de corte, e a coordenada $a_{i p}$ representa a quantidade de vezes que o item de comprimento $\ell_{i}$ aparece no p-ésimo padrão. Note que, para (1) satisfazer um padrão de corte, deve-se obedecer à seguinte restrição física:

$$
\ell_{1} a_{1 p}+\ell_{2} a_{2 p}+\ldots+\ell_{i} a_{i p}+\ldots+\ell_{I} a_{I p} \leq L, \quad i=1, . ., I, p=1, . ., P
$$

Nas inúmeras situações práticas podem ocorrer variações do problema. Nesse sentido, consideraremos que o estoque é composto por barras de vários comprimentos, cuja disponibilidade é limitada, entretanto, suficiente para o atendimento dos itens demandados. Para tanto, vamos considerar que o estoque de barras é composto por $J$ tipos de barras, de comprimentos $L_{1}, L_{2}, \ldots, L_{j}, \ldots, L_{J}$. Denotaremos por $e_{1}, e_{2}, \ldots, e_{j}, \ldots, e_{J}$ a quantidade disponível das respectivas

\footnotetext{
${ }^{2}$ Em problemas práticos, $I$ é da ordem de dezenas, enquanto a quantidade de maneiras de arranjos para a disposição das facas, que depende de $I, L$ e $\ell_{i}, i=1, \ldots, I$, pode ser da ordem de centenas de milhares.
} 
barras. Neste trabalho este problema será denominado Problema de Corte de Estoque Unidimensional Restrito (PCER). Matematicamente:

Minimizar:

$$
f(x)=\sum_{j=1}^{J} \sum_{p=1}^{P_{j}} T_{p j} x_{p j}
$$

Sujeito a:

$$
\begin{aligned}
& \sum_{j=1}^{J} \sum_{p=1}^{P_{j}} a_{p j} x_{p j}=\mathbf{d} \\
& \sum_{p=1}^{P_{j}} x_{p j} \leq e_{j}, j=1, \ldots, J \\
& x_{p j} \geq 0 \text { e inteiro, } p=1, \ldots, P_{j}, j=1, \ldots, J
\end{aligned}
$$

Em que, $\mathbf{a}_{p j}=\left(a_{1 p j}, a_{2 p j}, \ldots, a_{I p j}\right)^{T}$ é um vetor associado a um padrão de corte na j-ésima barra, $a_{1 p j}$ é a quantidade de vezes que o item de comprimento $\ell_{i}$ aparece no p-ésimo padrão de corte, na barra de comprimento $L_{j}$, sendo $e_{j}$ a quantidade disponível dessa barra em estoque. Por fim, $x_{p j}$ representa o total de barras que devem ser cortadas, segundo o p-ésimo padrão de corte. Ainda, $\mathbf{d}=\left(\mathbf{d}_{1}, \mathbf{d}_{2}, \ldots, \mathbf{d}_{i}, \ldots, \mathbf{d}_{I}\right)^{T}$ é o vetor de demandas, $T_{p j}=L_{j}-\sum_{i=1}^{I} \ell_{i} a_{i p j}$ representa a sobra produzida pelo p-ésimo padrão de corte na barra de comprimento $L_{j}$, em que $i=1, \ldots, I, j=1 \ldots, J$ e $p=1, \ldots, P_{j}$. Qualquer solução deste problema, cujos componentes sejam inteiros e não negativos, fornece uma solução factível para o problema.

São problemas de vasta aplicabilidade prática, cuja aparente simplicidade entra em contraste com a grande dificuldade de resolução por conta do elevado número de variáveis envolvidas e da restrição de integralidade destas variáveis. Tais problemas pertencem à classe NP-difícil ${ }^{3}$ (Garey e Johnson, 1979; Dyckhoff, 1990, citado por Poldi e Arenales, 2009) que, grosso modo, podemos dizer que são problemas improváveis de serem resolvidos num tempo polinomial aceitável. Em processos de corte, buscando uma melhor utilização da matéria-prima, muitas indústrias, ao invés de descartar as sobras do processo procuram utilizá-las, desde que apresentem condições para isto. O problema ao qual daremos ênfase neste trabalho é o Problema de Corte de Estoque Unidimensional com Aproveitamento de Sobras (PCES), que considera também as perdas (leiase sobras não aproveitáveis) e os retalhos (leia-se sobras aproveitáveis) obtidos em um processo de

\footnotetext{
${ }^{3}$ Polinomial Não Determinístico Difícil.
} 
corte. Adotaremos como retalho qualquer comprimento maior ou igual ao comprimento do menor item demandado.

No ponto de vista prático, a definição de perdas e retalhos é relativa. Um comprimento mínimo aceitável como retalho é um critério que deve ser estabelecido pela indústria. É possível considerar, por exemplo, o comprimento do menor item demandado, o comprimento do maior item demandado, a média dos comprimentos dos itens demandados, ou um comprimento qualquer préestabelecido pelo usuário. Na literatura, são vários os trabalhos que consideram como retalho qualquer comprimento maior ou igual ao comprimento do menor item demandado, a citar Pinto (2008), Farias (2011), Hoto e outros (2003), Gradisar e outros (1997), (1999a, b), Gradisar e Trkman (2005), Abuabara e Morabito (2008). É importante ressaltar que essa atribuição pode não ser interessante no caso em que o conjunto dos itens demandados inclui um item atípico de comprimento muito pequeno ou apenas itens de comprimentos grandes. Desse modo o processo de corte iria gerar, no primeiro caso, muitos retalhos pequenos e de pouco uso, ou, no segundo caso, muitas perdas que poderiam ser aceitáveis em processos futuros.

Partiremos do pressuposto que é mais desejável reduzir as perdas ao nível mínimo, e maximizar os retalhos ao nível máximo, de tal maneira que tais retalhos estejam concentrados na menor quantidade possível de padrões, desde que seja mantido o objetivo inicial de um problema de corte de estoque: minimizar sobras.

O principal objetivo deste trabalho consiste em determinar soluções inteiras para o PCES. Para isso faremos um estudo computacional dos modelos matemáticos propostos por Pinto (2008). A eficácia dos modelos será analisada pela comparação dos resultados com as de heurísticas propostas por Cherri e outros (2009). As simulações computacionais sugeriram que é possível propor uma variação dos modelos propostos por Pinto (2008), na tentativa de buscar soluções melhores que as já encontradas, o que também se constitui num objetivo a ser alcançado.

Para exemplificar, considere $J=2$ barras de comprimentos $L_{1}=170 \mathrm{~cm}$ e $L_{2}=100 \mathrm{~cm}$, cuja disponibilidade em estoque seja $e_{1}=2$ e $e_{2}=3$ respectivamente, para cortar $I=3$ tipos de itens de comprimentos $\ell_{1}=30 \mathrm{~cm}, \ell_{2}=50 \mathrm{~cm} \mathrm{e} \ell_{3}=55 \mathrm{~cm}$, de modo a atender às respectivas demandas $d_{1}=2, d_{2}=4$ e $d_{3}=3$. A Figura 3 ilustra três soluções factíveis para esse problema. 
Figura 3 - Representação dos dados do exemplo e três soluções factíveis, considerando o problema de corte de estoque com aproveitamento de sobras

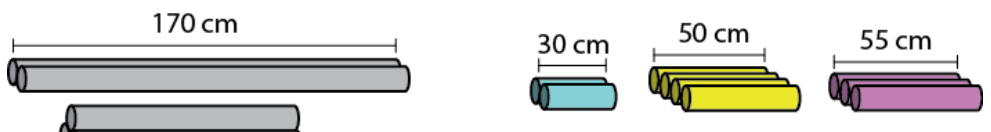

Solução 1

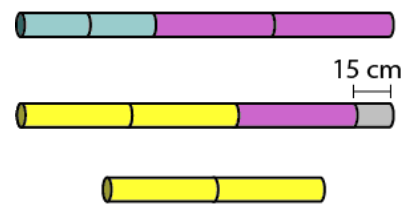

Perda total: $15 \mathrm{~cm}$ Retalho total: $0 \mathrm{~cm}$
Solução 2

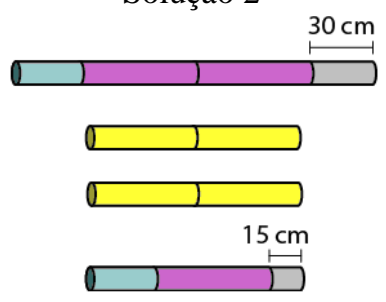

Perda total: $15 \mathrm{~cm}$ Retalho total: $30 \mathrm{~cm}$
Solução 3

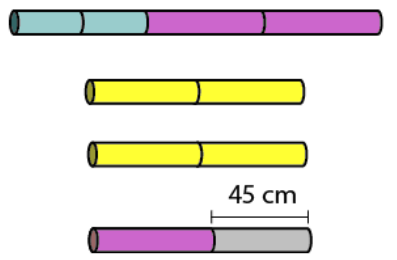

Perda total: $0 \mathrm{~cm}$ Retalho total: $45 \mathrm{~cm}$

Fonte: Autoria própria (2015)

Se considerarmos os problemas clássicos de corte com o objetivo de minimizar sobras, a solução 1 é mais desejável, e as soluções 2 e 3 são equivalentes. No entanto, se analisarmos pelo ponto de vista de um problema de corte de estoque com aproveitamento de sobras, a solução 2 é menos desejável que as outras duas, visto que apresenta uma perda total maior que a da solução 3 e igual à da solução 1, que por sua vez não gerou retalhos. A solução 3 é a mais desejável pelo fato de apresentar perda nula e concentrar o retalho em uma única barra. Em todo caso, não podemos afirmar que uma das soluções apresentadas é solução ótima do exemplo. Para isso, devemos resolver o PCES.

\section{Abordagens de Resolução}

Há várias técnicas especializadas na tentativa de solucionar um PCE, com abordagens diferentes, como busca, programação, relaxação, grafos e outras. A dificuldade na resolução de problemas deste tipo reside no fato de que cada uma delas apresenta características específicas, o que torna a definição de um método geral eficiente pouco provável.

\subsection{Heurísticas propostas por Cherri e outros (2009)}

Cherri e outros (2009) desenvolveram heurísticas construtivas e residuais específicas para resolver um PCES, com base em modificações realizadas em heurísticas clássicas conhecidas na literatura. Essas heurísticas são: Construtiva $\mathrm{FFD}_{\mathrm{R}}$, Construtiva Gulosa ${ }_{\mathrm{R}}$, Residual $\mathrm{FFD}_{\mathrm{R}}$, Residual Gulosa $_{\mathrm{R}}$ e Residual por Arredondamento Guloso $\left(\mathrm{RAG}_{\mathrm{R}}\right)$ - versões 1, 2 e 3. 
As heurísticas construtivas $\mathrm{FFD}_{\mathrm{R}}$ e Gulosa $a_{\mathrm{R}}$ foram desenvolvidas a partir de alterações nas heurísticas construtivas clássicas FFD e Gulosa, respectivamente. As Heurísticas Residuais FFD $_{\mathrm{R}} \mathrm{e}$ Gulosa $_{R}$ foram desenvolvidas com modificações nas heurísticas residuais FFD e Gulosa, respectivamente. Para essas heurísticas, o clássico problema de corte é resolvido com as restrições de integralidade relaxadas e, para obter uma solução inteira aproximada, as soluções contínuas são arredondadas e o problema residual resultante é resolvido pelas heurísticas construtivas $\mathrm{FFD}_{\mathrm{R}}$ ou Gulosa $a_{R}$. Por fim, as Heurísticas Residuais $\mathrm{RAG}_{\mathrm{R}}$ - versões 1,2 e 3 foram desenvolvidas realizando modificações nas heurísticas RAG - versões 1, 2 e 3 propostas por Poldi e Arenales (2009).

\subsection{Modelos propostos por Pinto (2008)}

Fundamentando-se a partir de uma modelagem do PCES, Pinto (2008), sugere em sua dissertação de mestrado que uma boa tática para obter o conjunto das soluções ótimas ou quase ótimas do PCER e, em seguida, buscar nesse conjunto soluções que maximizem as sobras, distribuindo-as na menor quantidade de barras. Seguindo este princípio, Pinto (2008) propôs três estratégias de resolução para o PCES que, numa primeira etapa encontra o menor comprimento que deve ser cortado de barras para o atendimento dos itens demandados e, numa segunda etapa, busca concentrar as perdas na menor quantidade de barras. $\mathrm{O}$ autor ainda propôs outras duas estratégias, visando resolver o PCES numa única etapa. As estratégias serão descritas a seguir.

Para isso considere as constantes:

- $\quad L_{j}$ : comprimento da j-ésima barra, $j=1, \ldots, J$;

- $T_{j}$ : Perda na j-ésima barra, $j=1, \ldots, J$;

- $\quad M \geq \max \left\{L_{j} \mid j=1, \ldots, J\right\}$

- $\quad \ell_{i}:$ comprimento do i-ésimo item, $i=1, \ldots, I$;

- $\quad d_{i}$ : demanda do i-ésimo item, $i=1, \ldots, I$;

- $S=\min \left\{\ell_{i} \mid i=1, \ldots, I\right\}:$ comprimento mínimo considerado como retalho;

- $\quad N$ : quantidade de barras escolhida na primeira etapa;

- $\quad R$ : quantidade de barras usadas para concentrar retalhos. 
E as variáveis:

- $\quad a_{i j}$ : quantidade de itens de índice $i=1, \ldots, I$ cortados da barra de índice $j=1, \ldots, J$;

$-y_{j}=\left\{\begin{array}{l}1, \text { se a barra de índice } j \text { é escolhida } \\ 0, \text { caso contrário }\end{array}\right.$

- $w_{j}=\left\{\begin{array}{l}1, \text { se a barra de índice } j \text { é escolhida para concentrar retalhos } \\ 0, \text { caso contrário }\end{array}\right.$.

Nas três primeiras estratégias, a etapa inicial é sempre a mesma. O modelo matemático desta etapa é:

Minimizar:

$$
\sum_{j=1}^{J} y_{j} L_{j}
$$

Sujeito a:

$$
\begin{aligned}
& \sum_{j=1}^{J} a_{i j}=d_{i}, i=1, \ldots, I \\
& \sum_{i=1}^{I} \ell_{i} a_{i j} \leq L_{j} y_{j}, j=1, \ldots, J \\
& a_{i j} \geq 0 \text { e inteiro, } i=1, \ldots, I, j=1 \ldots, J \\
& y_{j} \in\{0,1\}, j=1, \ldots, J
\end{aligned}
$$

A função objetivo (7) minimiza o comprimento total utilizado para atender a demanda. A restrição (8) garante que a quantidade de itens produzidos seja igual à demanda. A restrição (9) funciona da seguinte forma: se a j-ésima barra está sendo usada $\left(y_{j}=1\right)$ a mesma equivale a uma restrição de mochila, por outro lado, se a j-ésima barra não está sendo usada $\left(y_{j}=0\right)$ a restrição obriga que $a_{i j}=0, i=1, \ldots, I$. Por fim, as restrições (10) e (11) definem as variáveis do problema.

A etapa seguinte nas três primeiras estratégias consiste em determinar um conjunto de padrões que atenda a demanda diminuindo as sobras ao nível mínimo, gerando o mínimo de perdas e o máximo de retalhos, de tal maneira que estes retalhos estejam concentrados na menor quantidade de barras. 
O modelo matemático utilizado na Estratégia 1 é:

Minimizar:

$$
\sum_{j=1}^{N}\left(L_{j}-\sum_{i=1}^{I} \ell_{i} a_{i j}\right)
$$

Sujeito a:

$$
\begin{aligned}
& \sum_{j=1}^{N} w_{j}=R \\
& \sum_{j=1}^{N} a_{i j}=d_{i}, i=1, \ldots, I \\
& L_{j}-\sum_{i=1}^{I} \ell_{i} a_{i j} \leq w_{j} L_{j}+\left(1-w_{j}\right)(S-1), j=1, \ldots, N \\
& L_{j}-\sum_{i=1}^{I} \ell_{i} a_{i j} \geq w_{j} S, j=1, \ldots, N \\
& a_{i j} \geq 0 \text { e inteiro, } i=1, \ldots, I, j=1 \ldots, N \\
& w_{j} \in\{0,1\}, j=1, \ldots, N
\end{aligned}
$$

A função objetivo (12) minimiza as sobras do processo. A restrição (13) garante que a quantidade de barras utilizadas para concentrar os retalhos seja igual ao número $R$ previamente fixado. A restrição (14) garante que a quantidade de itens produzidos seja igual à demanda. As restrições (15) e (16) juntas, indicam para cada barra, que o comprimento remanescente do corte dos itens deve estar no intervalo $\left[S, L_{j}\right]$ quando $w_{j}=1$, isto é, a barra deve gerar retalhos, ou deve estar no intervalo $[0, S)$ quando $w_{j}=0$, isto é, a barra não deve gerar retalhos. Por fim, as restrições (17) e (18) definem as variáveis do problema.

O modelo matemático utilizado na Estratégia 2 é:

Minimizar:

$$
\sum_{j=1}^{N} T_{j}
$$

Sujeito a:

$$
\sum_{j=1}^{N} w_{j}=R
$$




$$
\begin{aligned}
& \sum_{j=1}^{N} a_{i j}=d_{i}, i=1, \ldots, I \\
& L_{j}-\sum_{i=1}^{I} \ell_{i} a_{i j} \leq L_{j}+\left(1-w_{j}\right) M, j=1, \ldots, N \\
& L_{j}-\sum_{i=1}^{I} \ell_{i} a_{i j} \geq S-\left(1-w_{j}\right) M, j=1, \ldots, N \\
& L_{j}-\sum_{i=1}^{I} \ell_{i} a_{i j} \leq T_{j}+w_{j} M, j=1, \ldots, N \\
& L_{j}-\sum_{i=1}^{I} \ell_{i} a_{i j} \geq T_{j}-w_{j} M, j=1, \ldots, N \\
& a_{i j} \geq 0 \text { e inteiro, } i=1, \ldots, I, j=1 \ldots, N \\
& T_{j} \geq 0 \text { e inteiro, } j=1 \ldots, N \\
& w_{j} \in\{0,1\}, j=1, \ldots, N
\end{aligned}
$$

A função objetivo (19) minimiza as perdas do processo. As restrições (20) e (21) são semelhantes às restrições (13) e (14) respectivamente. As restrições (22) e (23) juntas, indicam para cada barra, que o comprimento remanescente do corte dos itens deve gerar retalhos quando $w_{j}=1$, e ficam inativas quando $w_{j}=0$. As restrições (24) e (25) juntas, indicam para cada barra, que o comprimento remanescente do corte dos itens deve gerar perdas quando $w_{j}=0$, e ficam inativas quando $w_{j}=1$. Por fim, as restrições (26), (27) e (28) definem as variáveis do problema.

$\mathrm{Na}$ Estratégia 3, as restrições do modelo da primeira e da segunda etapa são parecidas, mas a função objetivo é bem diferente:

Maximizar:

$$
\sum_{j=N-(R-1)}^{N}\left(L_{j}-\sum_{i=1}^{I} \ell_{i} a_{i j}\right)
$$

Sujeito a:

$$
\begin{aligned}
& \sum_{j=1}^{N} a_{i j}=d_{i}, i=1, \ldots, I \\
& \sum_{i=1}^{I} \ell_{i} a_{i j} \leq L_{j}, \quad j=1, \ldots, N
\end{aligned}
$$




$$
a_{i j} \geq 0 \text { e inteiro, } i=1, \ldots, I, j=1 \ldots, N
$$

A função objetivo (29) maximiza as sobras do processo nas barras de índice $j=N-(R-1), \ldots, N$. A restrição (30) garante que a quantidade de itens produzidos seja igual à demanda. A restrição (31) equivale a uma restrição de mochila. Por fim, a restrição (32) define as variáveis do problema. Note que ao fazer $R=1$, optou-se por concentrar as sobras na última barra, e, neste caso, foi realizada uma ordenação decrescente das barras, ou seja, $L_{1} \leq \ldots \leq L_{N}$.

O modelo matemático utilizado na Estratégia 4 é:

Minimizar:

$$
\sum_{j=1}^{J} y_{j} L_{j}
$$

Sujeito a:

$$
\begin{aligned}
& \sum_{j=1}^{J} w_{j}=R \\
& \sum_{j=1}^{J} a_{i j}=d_{i}, i=1, \ldots, I \\
& L_{j}-\sum_{i=1}^{I} \ell_{i} a_{i j} \leq w_{j} L_{j}+\left(1-w_{j}\right)(S-1), j=1, \ldots, J \\
& L_{j}-\sum_{i=1}^{I} \ell_{i} a_{i j} \geq w_{j} S, j=1, \ldots, J \\
& a_{i j} \geq 0 \text { e inteiro, } i=1, \ldots, I, j=1 \ldots, J \\
& y_{j} \in\{0,1\}, w_{j} \in\{0,1\}, j=1, \ldots, J
\end{aligned}
$$

A função objetivo (33) minimiza o comprimento total cortado no processo. A restrição (34) garante que a quantidade de barras utilizadas para concentrar os retalhos seja igual ao número $R$ previamente fixado. A restrição (35) garante que a quantidade de itens produzidos seja igual à demanda. As restrições (36) e (37) são equivalentes às restrições (15) e (16), respectivamente e, juntas, indicam para cada barra, que o comprimento remanescente do corte dos itens deve estar no intervalo $\left[S, L_{j}\right]$ quando $w_{j}=1$, isto é, a barra deve gerar retalhos, ou deve estar no intervalo $[0, S)$ quando $w_{j}=0$, isto é, a barra não deve gerar retalhos. Por fim, as restrições (38) e (39) definem as variáveis do problema. 
O modelo matemático utilizado na Estratégia 5 é:

Minimizar:

$$
\sum_{j=1}^{J}\left(T_{j}+y_{j} L_{j}\right)
$$

Sujeito a:

$$
\begin{aligned}
& \sum_{j=1}^{J} w_{j}=R \\
& \sum_{j=1}^{J} a_{i j}=d_{i}, i=1, \ldots, I \\
& y_{j} L_{j}-\sum_{i=1}^{I} \ell_{i} a_{i j} \leq L_{j}+\left(1-w_{j}\right) M, j=1, \ldots, J \\
& y_{j} L_{j}-\sum_{i=1}^{I} \ell_{i} a_{i j} \geq S-\left(1-w_{j}\right) M, j=1, \ldots, J \\
& y_{j} L_{j}-\sum_{i=1}^{I} \ell_{i} a_{i j} \leq T_{j}+w_{j} M, j=1, \ldots, J \\
& y_{j} L_{j}-\sum_{i=1}^{I} \ell_{i} a_{i j} \geq T_{j}-w_{j} M, j=1, \ldots, J \\
& a_{i j} \geq 0 \text { e inteiro, } i=1, \ldots, I, j=1 \ldots, J \\
& T_{j} \geq 0 \text { e inteiro, } j=1 \ldots, J \\
& y_{j} \in\{0,1\}, w_{j} \in\{0,1\}, j=1, \ldots, J
\end{aligned}
$$

A função objetivo (40) minimiza a perda e o comprimento total cortado no processo. As restrições (41) e (42) são semelhantes às restrições (34) e (35)respectivamente. As restrições (43) e (44) juntas, indicam para cada barra, que o comprimento remanescente do corte dos itens deve gerar retalhos quando $w_{j}=1$, e ficam inativas quando $w_{j}=0$, agindo de forma similar às restrições (22) e (23), respectivamente. As restrições (45) e (46) juntas, indicam para cada barra, que o comprimento remanescente do corte dos itens deve gerar perdas quando $w_{j}=0$, e ficam inativas quando $w_{j}=1$, agindo de forma similar às restrições (24) e (25), respectivamente. 


\subsection{Reformulação da Estratégia 5 de Pinto (2008)}

Embora as soluções apresentadas por Pinto (2008) indiquem que é frutífera a estratégia de selecionar barras para o atendimento de itens demandados e, posteriormente, concentrar as perdas na menor quantidade possível destas barras, diversas soluções foram apresentadas como "a heurística não encontrou solução", "solução inviável" ou "o código falhou". Muito provavelmente tais resultados indicam erros nas implementações dos modelos.

Em princípio, um desdobramento imediato deste trabalho trata-se de revisar e avaliar o desempenho das estratégias propostas por Pinto (2008) para os milhares de exemplares extraídos de um gerador aleatório. As simulações realizadas mostraram que a Estratégia 5 se destacou em relação às outras quatro estratégias. A estratégia 1 não teve grandes resultados, e as outras estratégias tiveram seus bons resultados entre uns e outros. Por consequência, percebemos que é possível explorar uma reformulação do modelo matemático da Estratégia 5, que justificaremos a seguir.

Analisando novamente as restrições (43)-(46), para cada barra escolhida $\left(y_{j}=1\right)$, temos:

- Quando $w_{j}=1$, as restrições (43) e (44) visam gerar retalhos e as restrições (45) e (46) ficam inativas, para $i=1, \ldots, I, j=1 \ldots, J$;

$$
\begin{aligned}
& S \leq L_{j}-\sum_{i=1}^{I} \ell_{i} a_{i j} \leq L_{j} \\
& T_{j}-M \leq L_{j}-\sum_{i=1}^{I} \ell_{i} a_{i j} \leq T_{j}+M
\end{aligned}
$$

De fato, para $i=1, \ldots, I, j=1 \ldots, J$ :

$$
T_{j}-M \leq S \leq L_{j}-\sum_{i=1}^{I} \ell_{i} a_{i j} \leq L_{j} \leq T_{j}+M
$$

- Quando $w_{j}=0$, as restrições (43) e (44) ficam inativas e as restrições (45) e (46) visam gerar perdas para $i=1, \ldots, I, j=1 \ldots, J$.

$$
S-M \leq L_{j}-\sum_{i=1}^{I} \ell_{i} a_{i j} \leq L_{j}+M
$$




$$
T_{j} \leq L_{j}-\sum_{i=1}^{I} \ell_{i} a_{i j} \leq T_{j}
$$

De fato, para $i=1, \ldots, I, j=1 \ldots, J$ :

$$
S-M \leq S \leq L_{j}-\sum_{i=1}^{I} \ell_{i} a_{i j} \leq T_{j} \leq L_{j}+M
$$

Realizando algumas simulações e comparando os resultados obtidos da Estratégia 5 com os das Estratégias 1, 2, 3 e 4, pudemos observar que há uma grande dificuldade na definição dos critérios utilizados para avaliar uma boa solução. A ideia de "melhor" solução é relativa e depende, inicialmente, da decisão a ser tomada: minimizar sobras (Estratégia 1), minimizar perdas (Estratégia 2), maximizar as sobras em uma quantidade reduzida de barras (Estratégia 3), minimizar o comprimento total a ser cortado (Estratégia 4) ou uma combinação destas (Estratégia 5). Em nossa análise, acreditamos que do ponto de vista prático a indústria privilegia manter as perdas no seu patamar mínimo. Visando isso, para a reformulação da Estratégia 5 inicialmente eliminamos o termo $y_{j} L_{j}$ da função objetivo (40) que determina o comprimento total cortado. Desse modo, o objetivo passa a ser de minimizar perdas:

Minimizar:

$$
\sum_{j=1}^{J} T_{j}
$$

Em seguida, eliminamos o termo $T_{j}$ nas restrições (45) e (46):

$$
\begin{aligned}
& y_{j} L_{j}-\sum_{i=1}^{I} \ell_{i} a_{i j} \leq w_{j} M, j=1, \ldots, J \\
& y_{j} L_{j}-\sum_{i=1}^{I} \ell_{i} a_{i j} \geq-w_{j} M, j=1, \ldots, J
\end{aligned}
$$

Com tais alterações, quando $w_{j}=1$, as restrições (43) e (44) ainda visam gerar retalhos e as novas restrições (57) e (58) continuam inativas, para $i=1, \ldots, I, j=1 \ldots, J$.

$$
\begin{aligned}
& S \leq L_{j}-\sum_{i=1}^{I} \ell_{i} a_{i j} \leq L_{j} \\
& -M \leq L_{j}-\sum_{i=1}^{I} \ell_{i} a_{i j} \leq M
\end{aligned}
$$


De fato, para $i=1, \ldots, I, j=1 \ldots, J$ :

$$
-M \leq S \leq L_{j}-\sum_{i=1}^{I} \ell_{i} a_{i j} \leq L_{j} \leq M
$$

Por outro lado, quando $w_{j}=0$, as restrições (43) e (44) continuam inativas e as novas restrições (57) e (58) visam gerar perdas nulas para $i=1, \ldots, I, j=1 \ldots, J$.

$$
\begin{aligned}
& S-M \leq L_{j}-\sum_{i=1}^{I} \ell_{i} a_{i j} \leq L_{j}+M \\
& 0 \leq L_{j}-\sum_{i=1}^{I} \ell_{i} a_{i j} \leq 0
\end{aligned}
$$

De fato, para $i=1, \ldots, I, j=1 \ldots, J$ :

$$
S-M \leq 0 \leq L_{j}-\sum_{i=1}^{I} \ell_{i} a_{i j} \leq 0 \leq L_{j}+M
$$

Portanto, o modelo matemático Reformulado para a Estratégia 5 é:

Minimizar:

$$
\sum_{j=1}^{J} T_{j}
$$

Sujeito a:

$$
\begin{aligned}
& \sum_{j=1}^{J} w_{j}=R \\
& \sum_{j=1}^{J} a_{i j}=d_{i}, i=1, \ldots, I \\
& y_{j} L_{j}-\sum_{i=1}^{I} \ell_{i} a_{i j} \leq L_{j}+\left(1-w_{j}\right) M, j=1, \ldots, J \\
& y_{j} L_{j}-\sum_{i=1}^{I} \ell_{i} a_{i j} \geq S-\left(1-w_{j}\right) M, j=1, \ldots, J \\
& y_{j} L_{j}-\sum_{i=1}^{I} \ell_{i} a_{i j} \leq w_{j} M, j=1, \ldots, J \\
& y_{j} L_{j}-\sum_{i=1}^{I} \ell_{i} a_{i j} \geq-w_{j} M, j=1, \ldots, J \\
& a_{i j} \geq 0 \text { e inteiro, } i=1, \ldots, I, j=1 \ldots, J
\end{aligned}
$$




$$
\begin{aligned}
& T_{j} \geq 0 \text { e inteiro, } j=1 \ldots, J \\
& y_{j} \in[0,1], w_{j} \in[0,1], j=1, \ldots, J
\end{aligned}
$$

Sem perda de generalidade, chamaremos este modelo simplesmente de Estratégia 5 Reformulada. Ao analisar os resultados das simulações obtidas pela implementação desta estratégia, pudemos perceber que as soluções apresentaram perda nula e os retalhos são concentrados na menor quantidade possível de barras. Além disto, surpreendentemente a quantidade de barras utilizadas e/ou o comprimento total cortado foram em média menores ou iguais. Do ponto de vista prático, partimos do pressuposto que esta é a "melhor" solução para um PCES.

Para ilustrar, vamos considerar um PCES cujos dados são apresentados na Tabela 1.

\begin{tabular}{|c|c|c|c|c|c|c|}
\hline \multicolumn{7}{|c|}{ Barras } \\
\hline \multicolumn{2}{|r|}{$j$} & \multicolumn{3}{|c|}{$L_{j}$ (u.c.) } & \multicolumn{2}{|c|}{$e_{j}$} \\
\hline \multicolumn{2}{|r|}{1} & \multicolumn{3}{|c|}{1000} & \multicolumn{2}{|l|}{5} \\
\hline \multicolumn{2}{|r|}{2} & \multicolumn{3}{|c|}{1500} & \multicolumn{2}{|l|}{5} \\
\hline \multicolumn{2}{|r|}{3} & \multicolumn{3}{|c|}{2000} & \multicolumn{2}{|l|}{5} \\
\hline \multicolumn{2}{|r|}{4} & \multicolumn{3}{|c|}{2500} & \multicolumn{2}{|l|}{5} \\
\hline \multicolumn{7}{|c|}{ Itens } \\
\hline$i$ & $\ell_{i}$ (u.c.) & $d_{i}$ & & $i$ & $\ell_{i}$ (u.c.) & $d_{i}$ \\
\hline 1 & 240 & 8 & & 6 & 160 & 7 \\
\hline 2 & 210 & 7 & & 7 & 150 & 5 \\
\hline 3 & 250 & 8 & & 8 & 190 & 5 \\
\hline 4 & 120 & 9 & & 9 & 180 & 10 \\
\hline 5 & 170 & 7 & & 10 & 200 & 10 \\
\hline
\end{tabular}

Tabela 1 - Dados do exemplo.

Fonte: Dados aleatórios

Nos testes foram comparados quatro principais parâmetros (em quantidade ou em comprimento):

- Perda: barras que geram perdas;

- Retalho: barras que geram retalhos;

- Aprov.: em quantidade, representam barras que geram aproveitamento pleno, ou seja, perda nula; em comprimento, representam a demanda total atendida;

- Total: barras utilizadas no processo de corte.

Os resultados são apresentados na Tabela 2. 
Tabela 2 - Resultados do exemplo.

\begin{tabular}{ccccc|cccc}
\hline \multirow{2}{*}{ Estratégias } & \multicolumn{5}{c}{ Quantidade } & \multicolumn{4}{c}{ Comprimento (u.c.) } \\
\cline { 2 - 8 } & Perda & Retalho & Aprov. & Total & Perda & Retalho & Aprov. & Total \\
\hline 1 & 6 & 2 & 1 & 9 & 220 & 500 & 14280 & 15000 \\
2 & 5 & 1 & 3 & 9 & 320 & 400 & 14280 & 15000 \\
3 & 1 & 1 & 7 & 9 & 30 & 690 & 14280 & 15000 \\
4 & 5 & 2 & 3 & 10 & 200 & 520 & 14280 & 15000 \\
5 & 7 & 1 & 0 & 8 & 150 & 570 & 14280 & 15000 \\
5 Reformulada & 0 & 1 & 7 & 8 & 0 & 1220 & 14280 & 15500 \\
\hline
\end{tabular}

Fonte: Experimentos computacionais

Note que a Estratégia 5 Reformulada apresenta a solução com a menor perda e cujo retalho total é concentrado na menor quantidade possível de barras. De fato, a Estratégia 5 Reformulada selecionou a menor quantidade total de barras ( 8 barras), das quais nenhuma gera perda e apenas uma gera um único retalho de comprimento 1220 u.c. No entanto, o maior comprimento total cortado (15500 u.c.) deve-se à escolha de barras maiores para o atendimento da demanda.

Esses resultados podem ser justificados pelo fato de que a função objetivo busca minimizar a perda total ao mesmo tempo em que as restrições limitam a seleção de padrões de corte que apresentam perdas nulas ou retalhos. É provável que isto ocasione uma dominância do componente “Perdas" (nulas) sobre o componente "Retalhos", levando a resultados onde a perda é mínima (nula se for possível) e a quantidade de retalhos é concentrada na menor quantidade possível de barras. Por exemplo, a Estratégia 5 apresenta uma solução em que o retalho total de 570 u.c. é distribuída em 7 barras enquanto a Estratégia 5 Reformulada apresenta uma solução em que o retalho total de 1220 u.c. é distribuído em uma única barra.

\section{Experimentos Computacionais}

A quantidade de exemplares analisados no trabalho de Pinto (2008) é relativamente baixa se comparada aos trabalhos disponíveis na literatura. O autor selecionou oito exemplares: sete extraídos do trabalho de Abuabara (2006) e um extraído do trabalho de Sinuany-Stern e Weiner (1994). Resolvemos criar um próprio gerador de exemplares aleatórios, baseado no CUTGEN1 proposto por Gau e Wäscher (1995), variando-se a quantidade de itens, o tamanho dos itens e/ou as quantidades demandadas, dando atenção especial a problemas com baixa demanda, visto que estamos interessados em soluções boas e rápidas, mesmo que a otimalidade não seja garantida.

Os experimentos estão divididos em 12 classes de problemas, sendo que para cada classe foram gerados 100 exemplares. A seguir, a descrição dos critérios utilizados.

- Quantidade de barras: 4 barras padronizadas;

- Comprimento das barras: 1000 u.c., 1500 u.c., 2000 u.c. e 2500 u.c.; 
- Disponibilidade das barras: 5 cada;

- Quantidade de itens: 5, 10 ou 15;

- Comprimento dos itens: será gerado, aleatoriamente, dentro dos intervalos [50 u.c., 100 u.c.] para os itens de tamanho pequeno (P) e [100 u.c., 250 u.c.] para os itens de tamanho médio (M). Usando parâmetros que as aproximassem de situações reais, consideramos os comprimentos dos itens com dezenas inteiras;

- Demanda dos itens: será gerada, aleatoriamente, dentro dos intervalos $[1,5]$ para a demanda baixa (DB) e $[5,10]$ para a demanda média (DM);

- Comprimento para a sobra: menor item dentro do conjunto de itens do respectivo exemplar.

Obtemos, desta forma, problemas variando-se a quantidade de itens, o tamanho dos itens e/ou as quantidades demandadas. O código utilizado para caracterizar cada classe é formado por 5 dígitos: os dois primeiros representam a quantidade de itens (05, 10 ou 15); o próximo dígito representa o tamanho dos itens $(\mathrm{P}$ ou $\mathrm{M})$ e; os dois últimos dígitos que completam o código representam as demandas (DB ou DM), conforme a tabela 1. O código 15MDM, por exemplo, representa a classe com 15 itens de tamanho médio cujas demandas são médias.

\subsection{Resultados}

Os algoritmos das cinco estratégias apresentadas na Seção 2.2 e na Seção 2.3 foram implementados na linguagem de programação Mosel (Xpress-Mosel User Guide, 2008) e, utilizando o próprio Solver do programa, foram executados em um computador Intel Core i7 de 2,67 GHz com 12 GB de memória RAM. Quanto às heurísticas de Cherri e outros (2009), os exemplares selecionados foram executados pela professora Adriana Cristina Cherri Nicola, a quem registramos nossos sinceros agradecimentos.

Em razão da dificuldade em comparar as soluções do PCES, utilizaremos os seguintes critérios:

- Solução ideal: quando houver perda não nula em no máximo uma barra e todos os retalhos estiverem concentrados em no máximo uma barra;

- Solução aceitável: quando houver perda não nula em mais de uma barra ou todos os retalhos estiverem concentrados em mais de uma barra, não ambos; 
- Solução indesejável: quando houver perda não nula em mais de uma barra e todos os retalhos estiverem concentrados em mais de uma barra.

Nos testes foram comparados quatro principais parâmetros (em quantidade ou em comprimento):

- Perda: barras que geram perdas;

- Retalho: barras que geram retalhos;

- Aprov.: em quantidade, representam barras que geram aproveitamento pleno, ou seja, perda nula; em comprimento, representam a demanda total atendida;

- Total: barras utilizadas no processo de corte.

A eficácia dos métodos de resolução será analisada pela comparação dos resultados de uns com os outros. Os métodos obtiveram soluções factíveis para todos os exemplares gerados. As Tabelas 3 - 14 apresentam as médias para 100 exemplares de cada uma das 12 classes.

Tabela 3 - Resultados para os 100 exemplares da classe 1 - 5PDB

\begin{tabular}{|c|c|c|c|c|c|c|c|c|c|c|}
\hline \multirow{2}{*}{\multicolumn{2}{|c|}{ Métodos de resolução }} & \multirow{2}{*}{ Solução } & \multicolumn{4}{|c|}{ Quantidade } & \multicolumn{4}{|c|}{ Comprimento (u.c.) } \\
\hline & & & Perda & Retalho & Aprov. & Total & Perda & Retalho & Aprov. & Total \\
\hline \multirow{6}{*}{ Estratégias } & 1 & Ideal & 0,1 & 1,0 & 0,0 & 1,1 & 1,1 & 295,1 & 1113,8 & 1410,0 \\
\hline & 2 & Ideal & 0,0 & 1,0 & 0,0 & 1,1 & 0,9 & 295,3 & 1113,8 & 1410,0 \\
\hline & 3 & Ideal & 0,0 & 1,0 & 0,0 & 1,1 & 0,3 & 295,9 & 1113,8 & 1410,0 \\
\hline & 4 & Ideal & 0,0 & 1,0 & 0,0 & 1,1 & 1,5 & 294,7 & 1113,8 & 1410,0 \\
\hline & 5 & Ideal & 0,0 & 1,0 & 0,0 & 1,0 & 0,3 & 280,9 & 1113,8 & 1395,0 \\
\hline & 5 Ref. & Ideal & 0,0 & 1,0 & 0,1 & 1,1 & 0,0 & 581,2 & 1113,8 & 1695,0 \\
\hline \multirow{2}{*}{$\begin{array}{l}\text { Heurísticas } \\
\text { Construtivas }\end{array}$} & $\mathrm{FFD}_{\mathrm{R}}$ & Aceitável & 0,0 & 1,2 & 0,6 & 1,8 & 0,0 & 651,2 & 1113,8 & 1765,0 \\
\hline & Gulosa $_{\mathrm{R}}$ & Aceitável & 0,0 & 1,2 & 0,6 & 1,8 & 0,0 & 646,2 & 1113,8 & 1760,0 \\
\hline \multirow{5}{*}{$\begin{array}{c}\text { Heurísticas } \\
\text { Residuais }\end{array}$} & $\mathrm{FFD}_{\mathrm{R}}$ & Aceitável & 0,0 & 1,2 & 0,6 & 1,8 & 0,0 & 651,2 & 1113,8 & 1765,0 \\
\hline & Gulosa $_{\mathrm{R}}$ & Aceitável & 0,0 & 1,2 & 0,6 & 1,8 & 0,0 & 646,2 & 1113,8 & 1760,0 \\
\hline & $\mathrm{RAG}_{\mathrm{R}} 1$ & Ideal & 0,3 & 1,0 & 0,4 & 1,8 & 7,4 & 643,8 & 1113,8 & 1765,0 \\
\hline & $\mathrm{RAG}_{\mathrm{R}} 2$ & Ideal & 0,3 & 1,0 & 0,5 & 1,8 & 4,6 & 646,6 & 1113,8 & 1765,0 \\
\hline & $\mathrm{RAG}_{\mathrm{R}} 3$ & Ideal & 0,3 & 1,0 & 0,4 & 1,8 & 7,5 & 643,7 & 1113,8 & 1765,0 \\
\hline
\end{tabular}

Fonte: Experimentos computacionais 
Tabela 4 - Resultados para os 100 exemplares da classe 2 - 5PDM

\begin{tabular}{|c|c|c|c|c|c|c|c|c|c|c|}
\hline \multirow{2}{*}{\multicolumn{2}{|c|}{ Métodos de resolução }} & \multirow{2}{*}{ Solução } & \multicolumn{4}{|c|}{ Quantidade } & \multicolumn{4}{|c|}{ Comprimento (u.c.) } \\
\hline & & & Perda & Retalho & Aprov. & Total & Perda & Retalho & Aprov. & Total \\
\hline \multirow{6}{*}{ Estratégias } & 1 & Aceitável & 0,8 & 1,1 & 0,2 & 2,1 & 24,2 & 255,3 & 2810,5 & 3090,0 \\
\hline & 2 & Aceitável & 0,6 & 1,2 & 0,3 & 2,1 & 16,7 & 262,8 & 2810,5 & 3090,0 \\
\hline & 3 & Ideal & 0,2 & 1,0 & 0,9 & 2,1 & 2,6 & 276,9 & 2810,5 & 3090,0 \\
\hline & 4 & Aceitável & 0,6 & 1,2 & 0,2 & 2,1 & 17,3 & 262,2 & 2810,5 & 3090,0 \\
\hline & 5 & Ideal & 0,0 & 1,0 & 1,3 & 2,4 & 0,3 & 279,2 & 2810,5 & 3090,0 \\
\hline & 5 Ref. & Ideal & 0,0 & 1,0 & 1,1 & 2,1 & 0,0 & 1299,5 & 2810,5 & 4110,0 \\
\hline \multirow{2}{*}{$\begin{array}{l}\text { Heurísticas } \\
\text { Construtivas }\end{array}$} & $\mathrm{FFD}_{\mathrm{R}}$ & Aceitável & 0,0 & 1,2 & 2,1 & 3,3 & 0,0 & 559,5 & 2810,5 & 3370,0 \\
\hline & Gulosa $_{\mathrm{R}}$ & Aceitável & 0,0 & 1,2 & 2,2 & 3,3 & 0,0 & 569,5 & 2810,5 & 3380,0 \\
\hline \multirow{5}{*}{$\begin{array}{c}\text { Heurísticas } \\
\text { Residuais }\end{array}$} & $\mathrm{FFD}_{\mathrm{R}}$ & Aceitável & 0,0 & 1,2 & 2,1 & 3,3 & 0,9 & 558,6 & 2810,5 & 3370,0 \\
\hline & Gulosa $_{\mathrm{R}}$ & Aceitável & 0,0 & 1,1 & 2,1 & 3,3 & 0,9 & 568,6 & 2810,5 & 3380,0 \\
\hline & $\mathrm{RAG}_{\mathrm{R}} 1$ & Aceitável & 0,0 & 1,2 & 1,1 & 2,3 & 0,5 & 464,0 & 2810,5 & 3275,0 \\
\hline & $\mathrm{RAG}_{\mathrm{R}} 2$ & Aceitável & 0,0 & 1,2 & 1,1 & 2,3 & 0,5 & 469,0 & 2810,5 & 3280,0 \\
\hline & $\mathrm{RAG}_{\mathrm{R}} 3$ & Aceitável & 0,0 & 1,2 & 1,1 & 2,3 & 0,5 & 474,0 & 2810,5 & 3285,0 \\
\hline
\end{tabular}

Fonte: Experimentos computacionais

Tabela 5 - Resultados para os 100 exemplares da classe 3 - 5MDB

\begin{tabular}{|c|c|c|c|c|c|c|c|c|c|c|}
\hline \multirow{2}{*}{\multicolumn{2}{|c|}{ Métodos de resolução }} & \multirow{2}{*}{ Solução } & \multicolumn{4}{|c|}{ Quantidade } & \multicolumn{4}{|c|}{ Comprimento (u.c.) } \\
\hline & & & Perda & Retalho & Aprov. & Total & Perda & Retalho & Aprov. & Total \\
\hline \multirow{6}{*}{ Estratégias } & 1 & Ideal & 0,9 & 1,0 & 0,1 & 2,1 & 50,4 & 259,1 & 2630,5 & 2940,0 \\
\hline & 2 & Aceitável & 0,8 & 1,2 & 0,1 & 2,1 & 41,8 & 267,7 & 2630,5 & 2940,0 \\
\hline & 3 & Ideal & 0,2 & 1,0 & 0,8 & 2,1 & 3,0 & 306,5 & 2630,5 & 2940,0 \\
\hline & 4 & Aceitável & 0,8 & 1,1 & 0,2 & 2,0 & 44,0 & 265,5 & 2630,5 & 2940,0 \\
\hline & 5 & Ideal & 0,1 & 1,0 & 0,9 & 2,0 & 2,7 & 306,8 & 2630,5 & 2940,0 \\
\hline & 5 Ref. & Ideal & 0,0 & 1,0 & 0,8 & 1,8 & 0,0 & 714,5 & 2630,5 & 3345,0 \\
\hline \multirow{2}{*}{$\begin{array}{l}\text { Heurísticas } \\
\text { Construtivas }\end{array}$} & $\mathrm{FFD}_{\mathrm{R}}$ & Aceitável & 0,0 & 1,4 & 1,7 & 3,1 & 0,0 & 574,5 & 2630,5 & 3205,0 \\
\hline & Gulosa $_{\mathrm{R}}$ & Aceitável & 0,0 & 1,5 & 1,5 & 3,0 & 0,2 & 599,3 & 2630,5 & 3230,0 \\
\hline \multirow{5}{*}{$\begin{array}{c}\text { Heurísticas } \\
\text { Residuais }\end{array}$} & $\mathrm{FFD}_{\mathrm{R}}$ & Aceitável & 0,0 & 1,4 & 1,6 & 3,1 & 0,7 & 573,8 & 2630,5 & 3205,0 \\
\hline & Gulosa $_{\mathrm{R}}$ & Aceitável & 0,0 & 1,4 & 1,5 & 3,0 & 0,9 & 588,6 & 2630,5 & 3220,0 \\
\hline & $\mathrm{RAG}_{\mathrm{R}} 1$ & Aceitável & 0,1 & 1,3 & 1,0 & 2,5 & 3,4 & 561,1 & 2630,5 & 3195,0 \\
\hline & $\mathrm{RAG}_{\mathrm{R}} 2$ & Aceitável & 0,1 & 1,4 & 1,1 & 2,6 & 3,5 & 561,0 & 2630,5 & 3195,0 \\
\hline & $\mathrm{RAG}_{\mathrm{R}} 3$ & Aceitável & 0,1 & 1,3 & 1,0 & 2,5 & 3,4 & 561,1 & 2630,5 & 3195,0 \\
\hline
\end{tabular}

Fonte: Experimentos computacionais

Tabela 6 - Resultados para os 100 exemplares da classe 4-5MDM

\begin{tabular}{|c|c|c|c|c|c|c|c|c|c|c|}
\hline \multirow{2}{*}{\multicolumn{2}{|c|}{ Métodos de resolução }} & \multirow{2}{*}{ Solução } & \multicolumn{4}{|c|}{ Quantidade } & \multicolumn{4}{|c|}{ Comprimento (u.c.) } \\
\hline & & & Perda & Retalho & Aprov. & Total & Perda & Retalho & Aprov. & Total \\
\hline \multirow{6}{*}{ Estratégias } & 1 & Indesejável & 2,4 & 1,1 & 0,8 & 4,3 & 107,0 & 193,0 & 6710,0 & $\overline{7010,0}$ \\
\hline & 2 & Indesejável & 2,1 & 1,1 & 1,1 & 4,3 & 95,5 & 204,5 & 6710,0 & 7010,0 \\
\hline & 3 & Ideal & 0,3 & 1,0 & 3,0 & 4,3 & 3,3 & 296,7 & 6710,0 & 7010,0 \\
\hline & 4 & Indesejável & 2,1 & 1,2 & 0,9 & 4,1 & 91,4 & 208,6 & 6710,0 & 7010,0 \\
\hline & 5 & Aceitável & 1,5 & 1,0 & 1,9 & 4,3 & 38,1 & 261,9 & 6710,0 & 7010,0 \\
\hline & 5 Ref. & Ideal & 0,0 & 1,0 & 3,4 & 4,4 & 0,0 & 785,0 & 6710,0 & 7495,0 \\
\hline \multirow{2}{*}{$\begin{array}{l}\text { Heurísticas } \\
\text { Construtivas }\end{array}$} & $\mathrm{FFD}_{\mathrm{R}}$ & Aceitável & 0,1 & 1,5 & 4,9 & 6,4 & 0,6 & 754,4 & 6710,0 & 7465,0 \\
\hline & Gulosa $_{\mathrm{R}}$ & Aceitável & 0,1 & 1,5 & 4,8 & 6,3 & 0,6 & 739,4 & 6710,0 & 7450,0 \\
\hline \multirow{5}{*}{$\begin{array}{c}\text { Heurísticas } \\
\text { Residuais }\end{array}$} & $\mathrm{FFD}_{\mathrm{R}}$ & Aceitável & 0,0 & 1,5 & 4,3 & 5,8 & 0,6 & 689,4 & 6710,0 & 7400,0 \\
\hline & Gulosa $_{\mathrm{R}}$ & Aceitável & 0,0 & 1,5 & 4,3 & 5,8 & 0,4 & 704,6 & 6710,0 & 7415,0 \\
\hline & $\mathrm{RAG}_{\mathrm{R}} 1$ & Aceitável & 0,0 & 1,4 & 2,6 & 4,1 & 0,5 & 524,5 & 6710,0 & 7235,0 \\
\hline & $\mathrm{RAG}_{\mathrm{R}} 2$ & Aceitável & 0,0 & 1,4 & 2,6 & 4,0 & 0,5 & 514,5 & 6710,0 & 7225,0 \\
\hline & $\mathrm{RAG}_{\mathrm{R}} 3$ & Aceitável & 0,1 & 1,5 & 2,6 & 4,1 & 1,2 & 533,8 & 6710,0 & 7245,0 \\
\hline
\end{tabular}

Fonte: Experimentos computacionais 
Tabela 7 - Resultados para os 100 exemplares da classe 5-10PDB

\begin{tabular}{|c|c|c|c|c|c|c|c|c|c|c|}
\hline \multirow{2}{*}{\multicolumn{2}{|c|}{ Métodos de resolução }} & \multirow{2}{*}{ Solução } & \multicolumn{4}{|c|}{ Quantidade } & \multicolumn{4}{|c|}{ Comprimento (u.c.) } \\
\hline & & & Perda & Retalho & Aprov. & Total & Perda & Retalho & Aprov. & Total \\
\hline \multirow{6}{*}{ Estratégias } & 1 & Aceitável & 0,7 & 1,1 & 0,2 & 2,0 & 16,2 & 251,2 & 2252,6 & 2520,0 \\
\hline & 2 & Ideal & 0,6 & 1,0 & 0,4 & 2,0 & 13,1 & 254,3 & 2252,6 & 2520,0 \\
\hline & 3 & Ideal & 0,2 & 1,0 & 0,7 & 2,0 & 2,8 & 264,6 & 2252,6 & 2520,0 \\
\hline & 4 & Aceitável & 0,6 & 1,1 & 0,2 & 1,9 & 15,7 & 251,7 & 2252,6 & 2520,0 \\
\hline & 5 & Ideal & 0,0 & 1,0 & 0,7 & 1,7 & 0,0 & 267,4 & 2252,6 & 2520,0 \\
\hline & 5 Ref. & Ideal & 0,0 & 1,0 & 0,7 & 1,7 & 0,0 & 1007,4 & 2252,6 & 3260,0 \\
\hline \multirow{2}{*}{$\begin{array}{l}\text { Heurísticas } \\
\text { Construtivas }\end{array}$} & $\mathrm{FFD}_{\mathrm{R}}$ & Aceitável & 0,0 & 1,1 & 1,7 & 2,8 & 0,0 & 557,4 & 2252,6 & 2810,0 \\
\hline & Gulosa $_{\mathrm{R}}$ & Aceitável & 0,0 & 1,1 & 1,7 & 2,8 & 0,0 & 557,4 & 2252,6 & 2810,0 \\
\hline \multirow{5}{*}{$\begin{array}{c}\text { Heurísticas } \\
\text { Residuais }\end{array}$} & $\mathrm{FFD}_{\mathrm{R}}$ & Aceitável & 0,0 & 1,1 & 1,7 & 2,8 & 0,0 & 557,4 & 2252,6 & 2810,0 \\
\hline & Gulosa $_{\mathrm{R}}$ & Aceitável & 0,0 & 1,1 & 1,7 & 2,8 & 0,0 & 557,4 & 2252,6 & 2810,0 \\
\hline & $\mathrm{RAG}_{\mathrm{R}} 1$ & Aceitável & 0,0 & 1,1 & 1,1 & 2,2 & 0,0 & 527,4 & 2252,6 & 2780,0 \\
\hline & $\mathrm{RAG}_{\mathrm{R}} 2$ & Aceitável & 0,0 & 1,1 & 1,1 & 2,2 & 0,0 & 527,4 & 2252,6 & 2780,0 \\
\hline & $\mathrm{RAG}_{\mathrm{R}} 3$ & Aceitável & 0,0 & 1,1 & 1,1 & 2,2 & 0,0 & 522,4 & 2252,6 & 2775,0 \\
\hline
\end{tabular}

Fonte: Experimentos computacionais

Tabela 8 - Resultados para os 100 exemplares da classe 6 - 10PDM

\begin{tabular}{|c|c|c|c|c|c|c|c|c|c|c|}
\hline \multirow{2}{*}{\multicolumn{2}{|c|}{ Métodos de resolução }} & \multirow{2}{*}{ Solução } & \multicolumn{4}{|c|}{ Quantidade } & \multicolumn{4}{|c|}{ Comprimento (u.c.) } \\
\hline & & & Perda & Retalho & Aprov. & Total & Perda & Retalho & Aprov. & Total \\
\hline \multirow{6}{*}{ Estratégias } & 1 & Aceitável & 1,8 & 1,0 & 1,1 & 3,9 & 43,8 & 196,9 & 5594,3 & $\overline{5835,0}$ \\
\hline & 2 & Indesejável & 1,2 & 1,7 & 1,0 & 3,9 & 30,4 & 210,3 & 5594,3 & 5835,0 \\
\hline & 3 & Ideal & 0,2 & 1,0 & 2,7 & 3,9 & 2,5 & 238,2 & 5594,3 & 5835,0 \\
\hline & 4 & Indesejável & 1,6 & 1,4 & 0,7 & 3,7 & 19,6 & 201,1 & 5594,3 & 5815,0 \\
\hline & 5 & Ideal & 0,3 & 1,0 & 2,6 & 3,9 & 5,6 & 235,1 & 5594,3 & 5835,0 \\
\hline & 5 Ref. & Ideal & 0,0 & 1,0 & 3,3 & 4,3 & 0,0 & 905,7 & 5594,3 & 6500,0 \\
\hline \multirow{2}{*}{$\begin{array}{l}\text { Heurísticas } \\
\text { Construtivas }\end{array}$} & $\mathrm{FFD}_{\mathrm{R}}$ & Aceitável & 0,0 & 1,1 & 4,8 & 5,9 & 0,0 & 730,7 & 5594,3 & 6325,0 \\
\hline & Gulosa $_{\mathrm{R}}$ & Aceitável & 0,0 & 1,1 & 4,7 & 5,9 & 0,0 & 725,7 & 5594,3 & 6320,0 \\
\hline \multirow{5}{*}{$\begin{array}{c}\text { Heurísticas } \\
\text { Residuais }\end{array}$} & $\mathrm{FFD}_{\mathrm{R}}$ & Aceitável & 0,0 & 1,1 & 4,6 & 5,7 & 0,0 & 725,7 & 5594,3 & 6320,0 \\
\hline & Gulosa $_{\mathrm{R}}$ & Aceitável & 0,0 & 1,1 & 4,6 & 5,8 & 0,0 & 720,7 & 5594,3 & 6315,0 \\
\hline & $\mathrm{RAG}_{\mathrm{R}} 1$ & Aceitável & 0,0 & 1,2 & 2,3 & 3,4 & 0,0 & 470,7 & 5594,3 & 6065,0 \\
\hline & $\mathrm{RAG}_{\mathrm{R}} 2$ & Aceitável & 0,0 & 1,2 & 2,2 & 3,4 & 0,0 & 485,7 & 5594,3 & 6080,0 \\
\hline & $\mathrm{RAG}_{\mathrm{R}} 3$ & Aceitável & 0,0 & 1,2 & 2,2 & 3,4 & 0,0 & 495,7 & 5594,3 & 6090,0 \\
\hline
\end{tabular}

Fonte: Experimentos computacionais

Tabela 9 - Resultados para os 100 exemplares da classe 7 - 10MDB

\begin{tabular}{|c|c|c|c|c|c|c|c|c|c|c|}
\hline \multirow{2}{*}{\multicolumn{2}{|c|}{ Métodos de resolução }} & \multirow{2}{*}{ Solução } & \multicolumn{4}{|c|}{ Quantidade } & \multicolumn{4}{|c|}{ Comprimento (u.c.) } \\
\hline & & & Perda & Retalho & Aprov. & Total & Perda & Retalho & Aprov. & Total \\
\hline \multirow{6}{*}{ Estratégias } & 1 & Indesejável & 2,1 & 1,1 & 0,7 & 3,8 & 81,9 & 207,2 & 5315,9 & $\overline{5605,0}$ \\
\hline & 2 & Indesejável & 2,0 & 1,1 & 0,7 & 3,8 & 92,0 & 197,1 & 5315,9 & 5605,0 \\
\hline & 3 & Ideal & 0,4 & 1,0 & 2,4 & 3,8 & 3,3 & 285,8 & 5315,9 & 5605,0 \\
\hline & 4 & Indesejável & 2,1 & 1,1 & 0,6 & 3,8 & 100,2 & 188,9 & 5315,9 & 5605,0 \\
\hline & 5 & Ideal & 0,6 & 1,0 & 2,0 & 3,6 & 14,7 & 274,4 & 5315,9 & 5605,0 \\
\hline & 5 Ref. & Ideal & 0,0 & 1,0 & 2,5 & 3,5 & 0,0 & 839,1 & 5315,9 & 6155,0 \\
\hline \multirow{2}{*}{$\begin{array}{l}\text { Heurísticas } \\
\text { Construtivas }\end{array}$} & $\mathrm{FFD}_{\mathrm{R}}$ & Aceitável & 0,0 & 1,3 & 4,2 & 5,6 & 0,0 & 659,1 & 5315,9 & 5975,0 \\
\hline & Gulos $_{\mathrm{R}}$ & Aceitável & 0,0 & 1,3 & 4,1 & 5,5 & 0,0 & 629,1 & 5315,9 & 5945,0 \\
\hline \multirow{5}{*}{$\begin{array}{l}\text { Heurísticas } \\
\text { Residuais }\end{array}$} & $\mathrm{FFD}_{\mathrm{R}}$ & Aceitável & 0,0 & 1,4 & 4,2 & 5,5 & 0,0 & 659,1 & 5315,9 & 5975,0 \\
\hline & Gulos $_{\mathrm{R}}$ & Aceitável & 0,0 & 1,3 & 4,1 & 5,5 & 0,0 & 629,1 & 5315,9 & 5945,0 \\
\hline & $\mathrm{RAG}_{\mathrm{R}} 1$ & Aceitável & 0,0 & 1,3 & 2,2 & 3,5 & 0,3 & 513,8 & 5315,9 & 5830,0 \\
\hline & $\mathrm{RAG}_{\mathrm{R}} 2$ & Aceitável & 0,0 & 1,3 & 2,2 & 3,5 & 0,0 & 504,1 & 5315,9 & 5820,0 \\
\hline & $\mathrm{RAG}_{\mathrm{R}} 3$ & Aceitável & 0,0 & 1,3 & 2,2 & 3,5 & 0,1 & 509,0 & 5315,9 & 5825,0 \\
\hline
\end{tabular}

Fonte: Experimentos computacionais 
Tabela 10 - Resultados para os 100 exemplares da classe $8-10 \mathrm{MDM}$

\begin{tabular}{|c|c|c|c|c|c|c|c|c|c|c|}
\hline \multirow{2}{*}{\multicolumn{2}{|c|}{ Métodos de resolução }} & \multirow{2}{*}{ Solução } & \multicolumn{4}{|c|}{ Quantidade } & \multicolumn{4}{|c|}{ Comprimento (u.c.) } \\
\hline & & & Perda & Retalho & Aprov. & Total & Perda & Retalho & Aprov. & Total \\
\hline \multirow{6}{*}{ Estratégias } & 1 & Indesejável & 5,6 & 1,1 & 1,8 & 8,4 & 208,2 & 232,4 & 13264,4 & 13705,0 \\
\hline & 2 & Indesejável & 4,8 & 1,5 & 2,2 & 8,4 & 206,5 & 234,1 & 13264,4 & 13705,0 \\
\hline & 3 & Aceitável & 1,1 & 1,0 & 6,3 & 8,4 & 10,4 & 430,2 & 13264,4 & 13705,0 \\
\hline & 4 & Indesejável & 5,8 & 1,5 & 1,5 & 8,7 & 281,0 & 254,6 & 13264,4 & 13800,0 \\
\hline & 5 & Indesejável & 3,7 & 1,1 & 3,1 & 7,9 & 112,2 & 323,4 & 13264,4 & 13700,0 \\
\hline & 5 Ref. & Ideal & 0,0 & 1,0 & 7,0 & 8,0 & 0,0 & 960,6 & 13264,4 & 14225,0 \\
\hline \multirow{2}{*}{$\begin{array}{l}\text { Heurísticas } \\
\text { Construtivas }\end{array}$} & $\mathrm{FFD}_{\mathrm{R}}$ & Aceitável & 0,1 & 1,3 & 9,5 & 10,9 & 0,9 & 1099,7 & 13264,4 & 14365,0 \\
\hline & Gulosa $a_{R}$ & Aceitável & 0,1 & 1,4 & 9,4 & 10,8 & 0,8 & 1079,8 & 13264,4 & 14345,0 \\
\hline \multirow{5}{*}{$\begin{array}{c}\text { Heurísticas } \\
\text { Residuais }\end{array}$} & $\mathrm{FFD}_{\mathrm{R}}$ & Aceitável & 0,0 & 1,4 & 8,4 & 9,7 & 0,0 & 860,6 & 13264,4 & 14125,0 \\
\hline & Gulosa $a_{R}$ & Aceitável & 0,1 & 1,4 & 8,2 & 9,7 & 0,6 & 945,0 & 13264,4 & 14210,0 \\
\hline & $\mathrm{RAG}_{\mathrm{R}} 1$ & Aceitável & 0,0 & 1,3 & 5,6 & 7,0 & 0,0 & 560,6 & 13264,4 & 13825,0 \\
\hline & $\mathrm{RAG}_{\mathrm{R}} 2$ & Aceitável & 0,0 & 1,3 & 5,6 & 6,9 & 0,0 & 555,6 & 13264,4 & 13820,0 \\
\hline & $\mathrm{RAG}_{\mathrm{R}} 3$ & Aceitável & 0,0 & 1,4 & 5,6 & 7,1 & 0,1 & 580,5 & 13264,4 & 13845,0 \\
\hline
\end{tabular}

Fonte: Experimentos computacionais

Tabela 11 - Resultados para os 100 exemplares da classe 9-15PDB

\begin{tabular}{|c|c|c|c|c|c|c|c|c|c|c|}
\hline \multirow{2}{*}{\multicolumn{2}{|c|}{ Métodos de resolução }} & \multirow{2}{*}{ Solução } & \multicolumn{4}{|c|}{ Quantidade } & \multicolumn{4}{|c|}{ Comprimento (u.c.) } \\
\hline & & & Perda & Retalho & Aprov. & Total & Perda & Retalho & Aprov. & Total \\
\hline \multirow{6}{*}{ Estratégias } & 1 & Ideal & 0,9 & 1,0 & 0,3 & 2,3 & 21,0 & 239,7 & 3314,3 & 3575,0 \\
\hline & 2 & Aceitável & 0,7 & 1,2 & 0,5 & 2,3 & 15,1 & 245,6 & 3314,3 & 3575,0 \\
\hline & 3 & Ideal & 0,2 & 1,0 & 1,1 & 2,3 & 2,3 & 258,4 & 3314,3 & 3575,0 \\
\hline & 4 & Aceitável & 0,9 & 1,2 & 0,3 & 2,4 & 22,8 & 237,9 & 3314,3 & 3575,0 \\
\hline & 5 & Ideal & 0,0 & 1,0 & 1,8 & 2,8 & 0,0 & 260,7 & 3314,3 & 3575,0 \\
\hline & 5 Ref. & Ideal & 0,0 & 1,0 & 1,4 & 2,4 & 0,0 & 1210,7 & 3314,3 & 4525,0 \\
\hline \multirow{2}{*}{$\begin{array}{l}\text { Heurísticas } \\
\text { Construtivas }\end{array}$} & $\mathrm{FFD}_{\mathrm{R}}$ & Aceitável & 0,0 & 1,1 & 2,8 & 3,8 & 0,0 & 530,7 & 3314,3 & 3845,0 \\
\hline & Gulosa $_{\mathrm{R}}$ & Aceitável & 0,0 & 1,1 & 2,7 & 3,8 & 0,0 & 530,7 & 3314,3 & 3845,0 \\
\hline \multirow{5}{*}{$\begin{array}{c}\text { Heurísticas } \\
\text { Residuais }\end{array}$} & $\mathrm{FFD}_{\mathrm{R}}$ & Aceitável & 0,0 & 1,1 & 2,8 & 3,8 & 0,0 & 530,7 & 3314,3 & 3845,0 \\
\hline & Gulosa $_{\mathrm{R}}$ & Aceitável & 0,0 & 1,1 & 2,7 & 3,8 & 0,0 & 530,7 & 3314,3 & 3845,0 \\
\hline & $\mathrm{RAG}_{\mathrm{R}} 1$ & Aceitável & 0,0 & 1,2 & 1,3 & 2,4 & 0,0 & 485,7 & 3314,3 & 3800,0 \\
\hline & $\mathrm{RAG}_{\mathrm{R}} 2$ & Aceitável & 0,0 & 1,2 & 1,3 & 2,4 & 0,0 & 485,7 & 3314,3 & 3800,0 \\
\hline & $\mathrm{RAG}_{\mathrm{R}} 3$ & Aceitável & 0,0 & 1,2 & 1,3 & 2,4 & 0,0 & 480,7 & 3314,3 & 3795,0 \\
\hline
\end{tabular}

Fonte: Experimentos computacionais

Tabela 12 - Resultados para os 100 exemplares da classe 10 - 15PDM

\begin{tabular}{c|c|c|ccccc|cccc}
\hline \multirow{2}{*}{ Métodos de resolução } & \multirow{2}{*}{ Solução } & \multicolumn{5}{|c|}{ Quantidade } & \multicolumn{4}{c}{ Comprimento (u.c.) } \\
\cline { 4 - 9 } & 1 & Indesejável & 2,6 & 1,1 & 1,7 & 5,4 & 64,5 & 225,0 & 8390,5 & 8680,0 \\
& 2 & Indesejável & 2,1 & 1,9 & 1,4 & 5,4 & 56,8 & 232,7 & 8390,5 & 8680,0 \\
\multirow{3}{*}{ Estratégias } & 3 & Ideal & 0,3 & 1,0 & 4,1 & 5,4 & 3,5 & 286,0 & 8390,5 & 8680,0 \\
& 4 & Indesejável & 2,6 & 1,6 & 1,2 & 5,3 & 66,3 & 223,2 & 8390,5 & 8680,0 \\
& 5 & Aceitável & 1,1 & 1,0 & 3,2 & 5,3 & 17,5 & 272,0 & 8390,5 & 8680,0 \\
& $5 \mathrm{Ref}$ & Ideal & 0,0 & 1,0 & 4,7 & 5,7 & 0,0 & 1044,5 & 8390,5 & 9435,0 \\
\hline Heurísticas & $\mathrm{FFD}_{\mathrm{R}}$ & Aceitável & 0,0 & 1,1 & 6,7 & 7,8 & 0,1 & 849,4 & 8390,5 & 9240,0 \\
Construtivas & $\mathrm{Gulosa}_{\mathrm{R}}$ & Aceitável & 0,0 & 1,2 & 6,7 & 7,8 & 0,0 & 854,5 & 8390,5 & 9245,0 \\
\hline \multirow{3}{*}{ Heurísticas } & $\mathrm{FFD}_{\mathrm{R}}$ & Aceitável & 0,0 & 1,1 & 6,1 & 7,2 & 0,2 & 754,3 & 8390,5 & 9145,0 \\
Residuais & $\mathrm{Gulosa}_{\mathrm{R}}$ & Aceitável & 0,0 & 1,1 & 6,1 & 7,2 & 0,0 & 764,5 & 8390,5 & 9155,0 \\
& $\mathrm{RAG}_{\mathrm{R}} 1$ & Aceitável & 0,0 & 1,3 & 3,4 & 4,7 & 0,0 & 579,5 & 8390,5 & 8970,0 \\
& $\mathrm{RAG}_{\mathrm{R}} 2$ & Aceitável & 0,0 & 1,3 & 3,4 & 4,7 & 0,0 & 579,5 & 8390,5 & 8970,0 \\
& $\mathrm{RAG}_{\mathrm{R}} 3$ & Aceitável & 0,0 & 1,3 & 3,5 & 4,7 & 0,0 & 604,5 & 8390,5 & 8995,0 \\
\hline
\end{tabular}

Fonte: Experimentos computacionais 
Tabela 13 - Resultados para os 100 exemplares da classe 11 - 15MDB

\begin{tabular}{|c|c|c|c|c|c|c|c|c|c|c|}
\hline \multirow{2}{*}{\multicolumn{2}{|c|}{ Métodos de resolução }} & \multirow{2}{*}{ Solução } & \multicolumn{4}{|c|}{ Quantidade } & \multicolumn{4}{|c|}{ Comprimento (u.c.) } \\
\hline & & & Perda & Retalho & Aprov. & Total & Perda & Retalho & Aprov. & Total \\
\hline \multirow{6}{*}{ Estratégias } & 1 & Indesejável & 2,9 & 1,0 & 1,1 & 5,1 & 98,3 & 234,4 & 7857,3 & 8190,0 \\
\hline & 2 & Indesejável & 2,7 & 1,2 & 1,2 & 5,1 & 111,8 & 220,9 & 7857,3 & 8190,0 \\
\hline & 3 & Ideal & 0,5 & 1,0 & 3,6 & 5,1 & 4,7 & 328,0 & 7857,3 & 8190,0 \\
\hline & 4 & Indesejável & 3,0 & 1,2 & 1,0 & 5,2 & 136,5 & 186,2 & 7857,3 & 8180,0 \\
\hline & 5 & Aceitável & 1,3 & 1,0 & 2,6 & 5,0 & 31,1 & 301,6 & 7857,3 & 8190,0 \\
\hline & 5 Ref. & Ideal & 0,0 & 1,0 & 4,0 & 5,0 & 0,0 & 977,7 & 7857,3 & 8835,0 \\
\hline \multirow{2}{*}{$\begin{array}{l}\text { Heurísticas } \\
\text { Construtivas }\end{array}$} & $\mathrm{FFD}_{\mathrm{R}}$ & Aceitável & 0,0 & 1,2 & 6,2 & 7,4 & 0,2 & 842,5 & 7857,3 & 8700,0 \\
\hline & Gulosa $_{\mathrm{R}}$ & Aceitável & 0,0 & 1,3 & 6,0 & 7,4 & 0,1 & 852,6 & 7857,3 & 8710,0 \\
\hline \multirow{5}{*}{$\begin{array}{c}\text { Heurísticas } \\
\text { Residuais }\end{array}$} & $\mathrm{FFD}_{\mathrm{R}}$ & Aceitável & 0,0 & 1,2 & 6,1 & 7,4 & 0,2 & 857,5 & 7857,3 & 8715,0 \\
\hline & Gulosa $_{R}$ & Aceitável & 0,0 & 1,3 & 6,0 & 7,3 & 0,1 & 897,6 & 7857,3 & 8755,0 \\
\hline & $\mathrm{RAG}_{\mathrm{R}} 1$ & Aceitável & 0,0 & 1,4 & 3,2 & 4,6 & 0,1 & 567,6 & 7857,3 & 8425,0 \\
\hline & $\mathrm{RAG}_{\mathrm{R}} 2$ & Aceitável & 0,0 & 1,4 & 3,2 & 4,6 & 0,1 & 567,6 & 7857,3 & 8425,0 \\
\hline & $\mathrm{RAG}_{\mathrm{R}} 3$ & Aceitável & 0,0 & 1,4 & 3,3 & 4,6 & 0,1 & 567,6 & 7857,3 & 8425,0 \\
\hline
\end{tabular}

Fonte: Experimentos computacionais

Tabela 14 - Resultados para os 100 exemplares da classe $12-15 \mathrm{MDM}$

\begin{tabular}{c|c|c|cccc|cccc}
\hline \multirow{2}{*}{ Métodos de resolução } & \multirow{2}{*}{ Solução } & \multicolumn{5}{c|}{ Quantidade } & \multicolumn{4}{c}{ Comprimento (u.c.) } \\
\cline { 4 - 10 } & & Perda & Retalho & Aprov. & Total & Perda & Retalho & Aprov. & Total \\
\hline \multirow{6}{*}{ Estratégias } & 1 & Indesejável & 7,5 & 1,1 & 3,7 & 12,3 & 279,3 & 419,9 & 19815,8 & 20515,0 \\
& 2 & Indesejável & 7,6 & 1,8 & 2,9 & 12,3 & 342,3 & 351,9 & 19815,8 & 20510,0 \\
& 3 & Aceitável & 1,5 & 1,0 & 9,8 & 12,3 & 20,3 & 678,9 & 19815,8 & 20515,0 \\
& 4 & Indesejável & 8,3 & 1,7 & 2,5 & 12,4 & 391,6 & 297,6 & 19815,8 & 20505,0 \\
& 5 & Indesejável & 5,3 & 1,3 & 4,7 & 11,3 & 161,2 & 333,0 & 19815,8 & 20310,0 \\
& $5 \mathrm{Ref.}$ & Ideal & 0,0 & 1,0 & 10,5 & 11,5 & 0,0 & 844,2 & 19815,8 & 20660,0 \\
\hline Heurísticas & $\mathrm{FFD}_{\mathrm{R}}$ & Aceitável & 0,3 & 1,2 & 12,6 & 14,0 & 2,7 & 1071,5 & 19815,8 & 20890,0 \\
Construtivas & $\mathrm{Gulosa}_{\mathrm{R}}$ & Aceitável & 0,1 & 1,3 & 12,8 & 14,1 & 0,5 & 1088,7 & 19815,8 & 20905,0 \\
\hline \multirow{3}{*}{ Heurísticas } & $\mathrm{FFD}_{\mathrm{R}}$ & Aceitável & 0,0 & 1,3 & 11,6 & 12,9 & 0,2 & 819,0 & 19815,8 & 20635,0 \\
Residuais & $\mathrm{Gulosa}_{\mathrm{R}}$ & Aceitável & 0,0 & 1,3 & 11,5 & 12,9 & 0,1 & 869,1 & 19815,8 & 20685,0 \\
& $\mathrm{RAG}_{\mathrm{R}} 1$ & Aceitável & 0,0 & 1,4 & 8,7 & 10,1 & 0,0 & 599,2 & 19815,8 & 20415,0 \\
& $\mathrm{RAG}_{\mathrm{R}} 2$ & Aceitável & 0,0 & 1,4 & 8,7 & 10,1 & 0,0 & 594,2 & 19815,8 & 20410,0 \\
& $\mathrm{RAG}_{\mathrm{R}} 3$ & Aceitável & 0,0 & 1,5 & 8,8 & 10,3 & 0,0 & 629,2 & 19815,8 & 20445,0 \\
\hline
\end{tabular}

Fonte: Experimentos computacionais

\section{Discussões}

Buscamos com este trabalho determinar soluções inteiras para um Problema de Corte de Estoque com Aproveitamento de Sobras (PCES) por meio de um estudo computacional dos modelos matemáticos propostos por Pinto (2008). Exploramos também uma reformulação do modelo matemático da Estratégia 5, a qual denotamos por Estratégia 5 Reformulada (5R).

A eficácia dos modelos foi analisada pela comparação dos resultados com os resultados de heurísticas propostas por Cherri e outros (2009), nos quais procedimentos heurísticos clássicos da literatura (construtivos e residuais) que resolvem problemas de corte de estoque foram modificados com a finalidade de resolverem problemas de corte de estoque com sobras aproveitáveis. Para isto, propomos um gerador aleatório de classes de exemplares, variando-se a quantidade de itens, o 
tamanho dos itens e/ou as quantidades demandadas, dando atenção especial a problemas com baixa demanda.

Com relação à identificação das melhores soluções, percebemos que, embora todos os métodos de resolução apresentem soluções factíveis, satisfatórias e pouco discrepantes em alguns casos, a escolha do melhor método de resolução para um PCES não é trivial, pois envolve a análise simultânea de diversos critérios. De acordo com os critérios utilizados para definir uma solução ideal, aceitável ou indesejada, conforme as classes vão crescendo em termos de dimensão e demanda dos exemplares, a concentração dos melhores resultados tende para a Estratégia 5R e para os procedimentos heurísticos, em particular para os residuais $R_{A G} 1, R_{R} G_{R} 2$ e $R A G_{R} 3$. De fato, embora para os exemplares da classe 5PDB as Estratégias 1, 2, 3, 4 e 5 apresentam um bom aproveitamento em relação ao total cortado, os resultados evidenciaram o contrário, à medida que analisamos as classes seguintes.

Em todas as classes estudadas os resultados da Estratégia 5R melhoram a superioridade já salientada, pois selecionam um número reduzido de barras, não geram perdas e concentram os retalhos em apenas uma barra.

Como perspectivas futuras de continuidade e melhoria deste trabalho, podemos citar:

- Uma variação dos modelos propostos por Pinto (2008), na tentativa de buscar soluções melhores que as já encontradas;

- Estender os modelos para uma abordagem por geração de colunas;

- Testar os métodos de resolução, por meio de exemplares com comprimentos e demandas maiores, e verificar como se comportam as soluções;

- Investigar, na literatura, trabalhos que tratam do problema de corte de estoque com aproveitamento de sobras para o caso bidimensional.

\section{Abstract}

A Cutting Stock Problem (CSP) basically consists of cut a set of larger parts (raw material) available in stock with the aim of produce smaller parts, optimizing a specific objective, which can minimize the amount of raw material to be cut, as well as the generated leftovers by the process or the cost associated to cut; maximize the profit, among others. In this context, the Cutting Stock Problem Using the Waste (CSPUW) focuses that question aiming at the better use of raw material, that is, use the waste in a future cutting process, as long as they offer conditions. What we seek, in this work, find full solutions to a CSPUW by means of computational studies of mathematical models proposed by Pinto (2008). We also explored a reformulation of the mathematical model of Strategy 5, by Pinto (2008), which we call Reformulated Strategy 5 (5R). We analyzed the efficiency of the models, considering avaliation criteria established by Cherri et al (2009), by computational simulations realized in a set of classes of pieces randomly generated. The results 
show that, with the increase of classes in terms of extent and demand of pieces, the 5R strategy shows better results to heuristic procedure, specially the residual RAGR1, RAGR2 e RAGR3.

Key-words: cutting stock problem; exploited of heuristic waste; random generator; inner linear programming problem.

\section{Referências}

ABUABARA, A. Otimização no corte de tubos estruturais: aplicação na indústria aeronáutica agrícola. 2006. 144 f. Dissertação (Mestrado em Engenharia de Produção) - Programa de Pós-Graduação em Engenharia de Produção, Universidade de São Carlos, São Carlos, 2006.

ABUABARA, A., MORABITO R., Modelos de programação inteira mista para o planejamento do corte unidimensional de tubos metálicos na indústria aeronáutica agrícola. Gestão e Produção, v. 15, set./dez. 2008. Disponível em: <http://www.scielo.br/scielo.php?script=sci_arttext\&pid=S0104-530X2008000300014〉. Aceso em: 31 jan. 2015.

CHERRI, A. C., ARENALES, N., YANASSE, H. H., The one dimensional cutting stock problems with usable leftover: A heuristic approach. European Journal of Operational Research, v. 196, maio/abr. 2009. crossref

CUI, Y., YANG, Y., A heuristic for the one-dimensional cutting stock problem with usable leftover. European Journal of Operational Research, v. 204, jul./out. 2010. crossref

DASH OPTIMIZATION, Xpress-Mosel User Guide, 2008.

DYCKHOFF, H. A typology of cutting and packing problems. European Journal Operational Reserarch, v. 44, 1990.

GAREY, M. R., JOHNSON, D. S., Computers and intractability: a guide to the NP-completeness. W. H., Freeman, 1979.

GAU, T., WÄSCHER, CUTGEN: A problem generator for the standard one-dimensional cutting stock problem. European Journal Operational Reserarch, v. 84, 1995.

GILMORE, P. C., GOMORY, R. E., A linear programming approach to the cutting stock problem - Part II. Operations Research, v. 11, nov./dez/ 1963. Disponível em:

<http://www.jstor.org/discover/10.2307/167827?sid=21105218882281\&uid=3737664\&uid=4\&uid=2> Acesso em 31 jan. 2015.

GRADISAR, M., JESENKO, J., RESINOVIC, C., Optimization of roll cutting in clothing industry. Computers \& Operational Reserarch, v. 101997.

GRADISAR, M., KLJAJIC, M., RESINOVIC, C., JESENKO, J., A sequential heuristic procedure for one-dimentional cutting. European Journal of Operational Reserarch, v. 114, 1999.

GRADISAR, M., RESINOVIC, C., KLJAJIC, M., A hybrid approach for optimization of one-dimentional cutting. European Jounal of Operational Research, v. 119, 1999.

GRADISAR, M., TRKMAN, P., A combined approach to the solution to the general one-dimentional cutting stock problem. Computers \& Operational Reserarch, v. 32, 2005.

HOTO, R. S. V., MACUlAN, N.; MARQUES, F., ARENALES, M. N., Um problema de corte com padrões compartimentados. Pesquisa Operacional, Rio de Janeiro, v. 23, n. 1, jan./abr. 2003. Disponível em: <http://dx.doi.org/10.1590/S0101-74382003000100013>. Acesso em: 31 jan. 2015.

PINTO, T. S., Uma proposta para resolver o problema de corte de estoque unidimensional com reaproveitamento de sobras por meio de dois objetivos. 68 f. Dissertação (Mestrado em Matemática Aplicada e Computacional) Programa de Pós-Graduação em Matemática Aplicada e Computacional, Universidade Estadual de Londrina, Londrina, 2008. 
POLDI, K. C., ARENALES, M. N., Heuristics for the one-dimensional cutting stock problem with limited multiple stock lengths. Computers and Operations Research, v. 36, n. 6, jun./jun., 2009. crossref

SINUANY-STERN, Z., WEINER I., The one dimensional cutting stock problem using two objectives. Journal of Operations Research Society, v. 45, 1994.

WÄSCHER, G., HAUßNER, H., SCHUMANN, H., An improved typology cutting and packing problems. European Journal of Operational Research, v. 183, set./dez. 2007. crossref

\section{Dados dos autores}

Nome completo: Eduardo Aparecido da Rosa Neto

Filiação Institucional: Universidade Estadual de Londrina - UEL

Endereço completo para correspondência: Rua Eliane Alvin Dias, 344, Jardim Império do Sol, Londrina, PR, CEP: 86073-770

Telefone para contato: (43) 3304-4574

e-mail: eduardo.neto@ensinolivre.com.br

Nome completo: Robinson Samuel Vieira Hoto

Filiação Institucional: Universidade Estadual de Londrina - UEL

Endereço completo para correspondência: Universidade Estadual de Londrina - UEL, Centro de Ciências Exatas, Departamento de Matemática - SimuLab (Lab Simulação e Otimização Sistemas), Rodovia Celso Garcia Cid, km 380, 344, Campus Universitário, Londrina, PR, CEP: 86057-970

Telefone para contato: (43) 3371-4150

e-mail: hoto@uel.br

Submetido: 05-03-2015

Aceito:14-09-2015 